\title{
Editorial
}

\section{Condensed Matter in Energy, Environment, and Beyond}

\author{
Hong Fang $\mathbb{D}^{1}{ }^{1}$ Jiajie Zhu, ${ }^{2}$ and Juncheng Zhang $^{3}$ \\ ${ }^{1}$ Department of Physics, Virginia Commonwealth University, Richmond, VA, USA \\ ${ }^{2}$ Department of Materials Science, Shenzhen University, Shenzhen, China \\ ${ }^{3}$ Department of Physics, Qingdao University, Qingdao, China \\ Correspondence should be addressed to Hong Fang; hfangtom@gmail.com
}

Received 7 April 2019; Accepted 7 April 2019; Published 2 May 2019

Copyright (C) 2019 Hong Fang et al. This is an open access article distributed under the Creative Commons Attribution License, which permits unrestricted use, distribution, and reproduction in any medium, provided the original work is properly cited.

One of the most important themes of today's scientific research is the design and development of energetic and environmental materials, as well as the understanding of their physics and chemistry. Condensed matters, such as organic-inorganic hybrid perovskites, superionic conductors, metal-organic frameworks, covalent organic frameworks, black phosphorus, penta-graphene, and mechanoluminescent materials, have attracted tremendous interest. In this special issue, we intend to cover recent theoretical and experimental progress in the condensed matter materials that have applications related to energy and environment, but also with a much broader touch on other important functionality. The focus will be on the physical properties of novel condensed matter materials in terms of their applications, for example, in solar cells, rechargeable batteries based on metal ions, greenhouse gas sequestration and conversion, gas sensory and separation, photovoltaics, photoluminescence, and mechanical sensors. The purpose of this special issue is to present the recent progress in these exciting fields. A brief summary of all accepted papers is provided below.

In the paper by S. Chen et al., they have reported the controlling physical surface structure of polyvinyl butyral (PVB) films by different film-forming methods, spin-coating, bar-coating, and electrospinning. The wettability of these PVB films was examined, and the surface morphologies and roughness were investigated. The results indicated that coating PVB films were hydrophilic, while electrospun films were hydrophobic. The physical surface structure was the key role on the interesting transition of their surface wettability. These results may help to find the way to control the PVB film surface wettability and then guide for applications.

The paper by G. P. Podust et al. presents their results of the dose dependencies of the light sum accumulation in $\mathrm{ZnSe}$ single crystals at a different X-ray excitation at $85 \mathrm{~K}$. The values of light sum accumulation at different deep traps were determined from the thermally stimulated luminescence and conductivity curves. It was confirmed that the accumulated light sum is uniquely determined by the dose of radiation only, that is, the product of the intensity of excitation by the time of X-ray exposure.

The work by M. Ambrožič and S. Kralj studied the impact of the cell thickness on configurations of line disclinations within a plane-parallel nematic cell. The LebwohlLasher semimicroscopic approach was used and (meta)stable nematic configurations were calculated using Brownian molecular dynamics. Defect patterns were enforced topologically via boundary conditions. The resulting structures exhibit line defects which either connect the facing plates or remain confined within the layers near confining plates. The first structure is stable in relatively thin cells and the latter one in thick cells. It is demonstrated that "history" of samples could have strong impact on resulting nematic configurations.

The paper by J. Burunkova et al. reported dodecanethiolfunctionalized gold nanoparticles that were incorporated into the polymer matrix of diurethane-methacrylate monomer mixture in the presence of $\mathrm{SiO}_{2}$ nanoparticles (8 wt\%) and a new reactive organic luminescent material ACAIN (1-N-(2-acryloyloxy-3-chloroprop-1-yl)-amino-5-isocyanonaphthalene, $1 \mathrm{wt} \%)$. It was shown that the addition of $\mathrm{SiO}_{2}$ nanoparticles enhances the fluorescent intensity of ACAIN by 1.5 times in the $450-550 \mathrm{~nm}$ spectral range. Furthermore, an additional fluorescence enhancement up to 4.5 times was observed when $\mathrm{Au}$ nanoparticles were added to the nanocomposite in a rather low $(0.12 \mathrm{wt} \%)$ concentration. 
The paper by E. Barrios-Salgado et al. reported PbS thin films with thickness between 100 and $150 \mathrm{~nm}$ which were grown for the first time by microwave-assisted chemical bath deposition in a commercial automated system with deposition times not exceeding $5 \mathrm{~min}$. X-ray diffraction analysis shows that the thin films have cubic rock salt type structure with good crystallinity. The grain size increased from 18 to $20 \mathrm{~nm}$, as the deposition time increased. Energy dispersive X-ray results confirm that the films are stoichiometric. Optical measurements show that thin films have relatively high absorption coefficients between 104 and 105 $\mathrm{cm}-1$ in the visible range. In addition, the films exhibit a direct gap, within the energy range from 1.0 to $1.35 \mathrm{eV}$. The electrical properties, such as conductivity, the Seebeck coefficient, carrier concentration, and carrier mobility, are discussed.

The paper by $\mathrm{D}$. Jiang et al. studied the effect of adding Co on crystallization behavior and magnetoimpedance effect of amorphous/nanocrystalline $\mathrm{FeCuNbSiB}$ alloy strips.

A. Teklu et al. studied stiffness, hardness, and the reduced Young's modulus of reduced graphene oxide by using nanoindentation coupled with atomic force microscopy.

The paper by N. D. Quan et al. reported the synthesis of lead-free $\mathrm{Bi}_{0.5}\left(\mathrm{Na}_{0.80} \mathrm{~K}_{0.20}\right)_{0.5} \mathrm{TiO}_{3}$ (BNKT) ferroelectric films on $\mathrm{Pt} / \mathrm{Ti} / \mathrm{SiO} 2 / \mathrm{Si}$ substrates via chemical solution deposition. The influence of crystallization time on the microstructures and ferroelectric and energy-storage properties of the films was investigated in detail. The obtained results indicate that the BNKT films have application potentials in advanced capacitors.

The paper by $\mathrm{H}$. Wang et al. reported the preparation of a lithium-rich manganese-based cathode material, $\mathrm{Li}_{1.25} \mathrm{Ni}_{0.2} \mathrm{Co}_{0.333} \mathrm{Fe}_{0.133} \mathrm{Mn}_{0.333} \mathrm{O}_{2}$, using a polyvinyl alcohol(PVA-) auxiliary sol-gel process using $\mathrm{MnO}_{2}$ as a template. The effect of the PVA content on the electrochemical properties and morphology of the material was investigated.

D. U. Combari et al. conducted an experiment and investigated the performance of a silicon photovoltaic module subjected to a magnetic field. The current-voltage and powervoltage characteristics were plotted in the same axis system and allowed us to find, as a function of the magnetic field, the electrical parameters of the photovoltaic module such as maximum electric power, fill factor, conversion efficiency, and charge resistance at the maximum power point. These electrical parameters were then used to calculate the series and shunt resistances of the equivalent circuit of the photovoltaic module. The results have shown that the efficiency of a solar module is affected by the presence of magnetic fields.

\section{Conflicts of Interest}

The authors declare that they have no conflict of interest.

\section{Acknowledgments}

We would like to express our gratitude to all authors who made this special issue possible. We hope this collection of articles will be useful to the scientific community. 

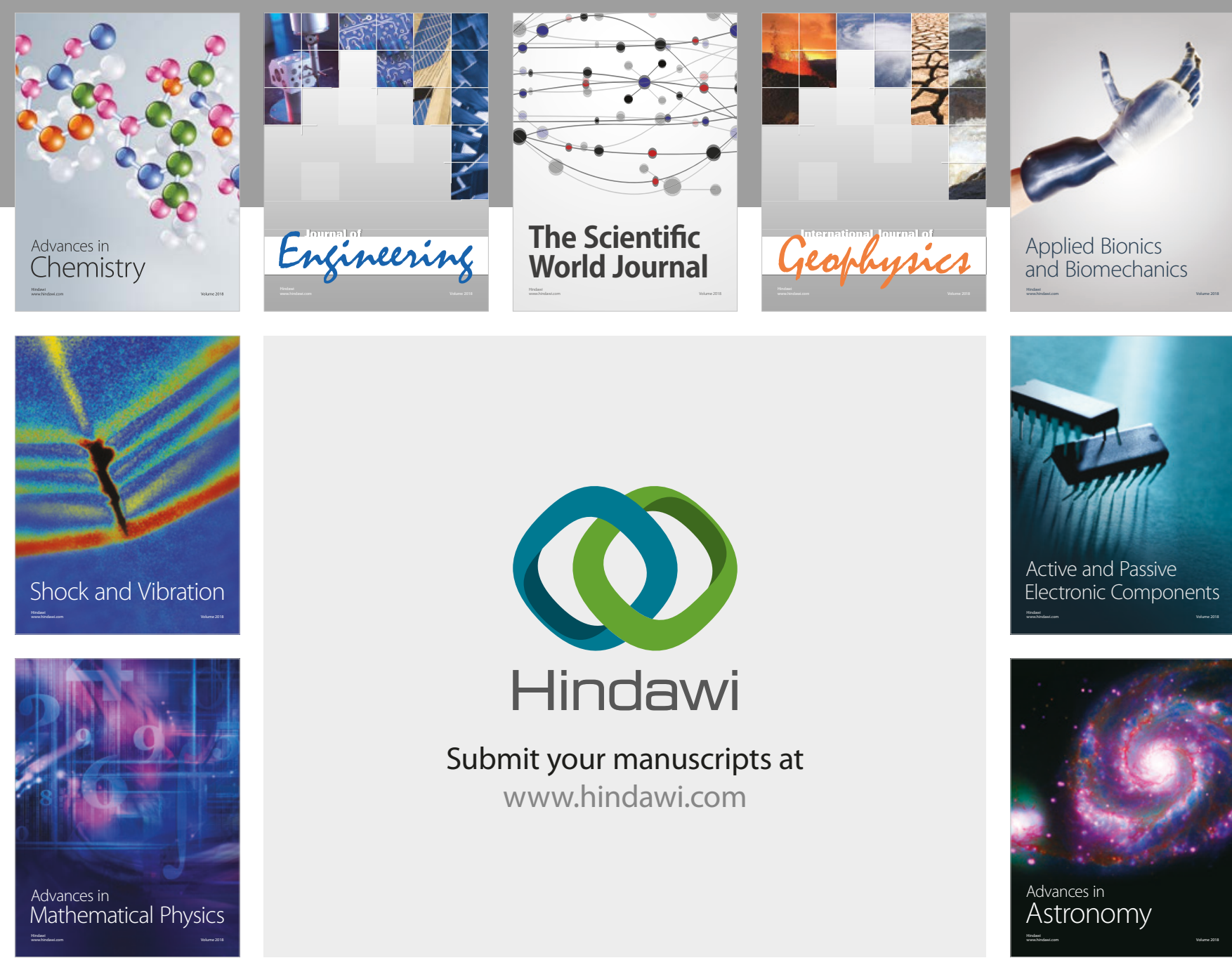

Submit your manuscripts at

www.hindawi.com

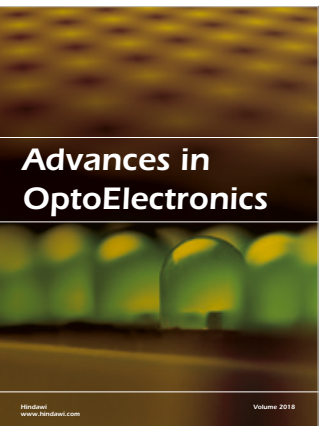

\section{Rotcting Machinery}
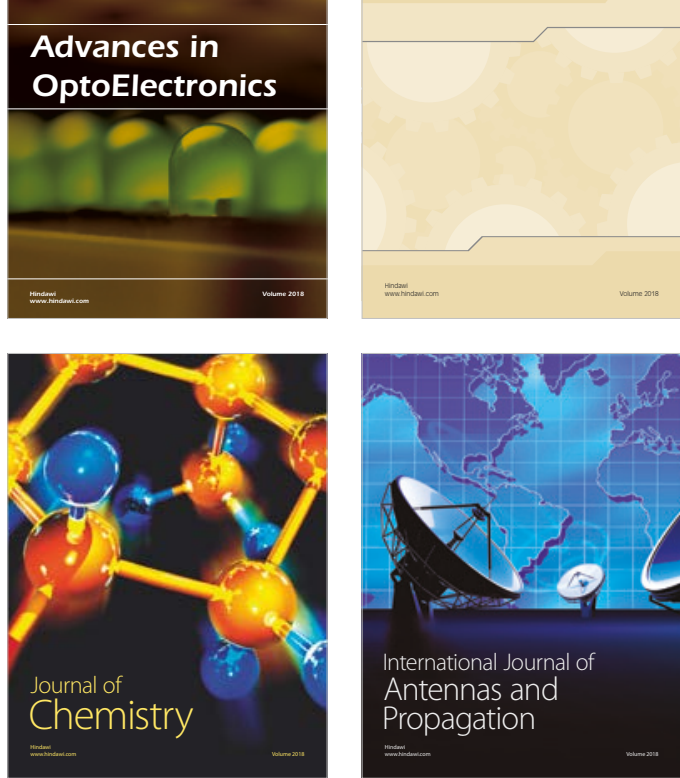

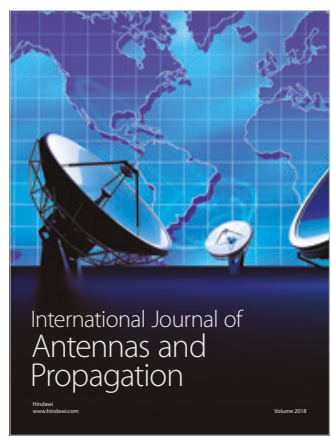

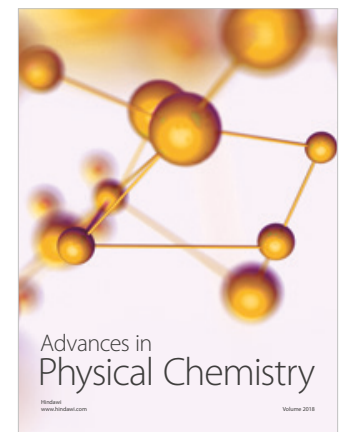

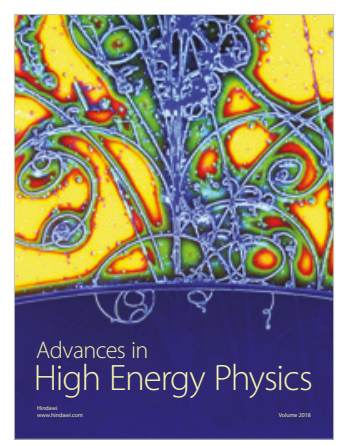

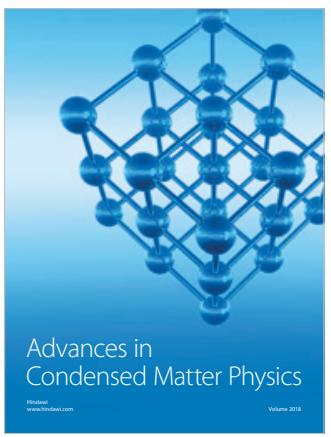

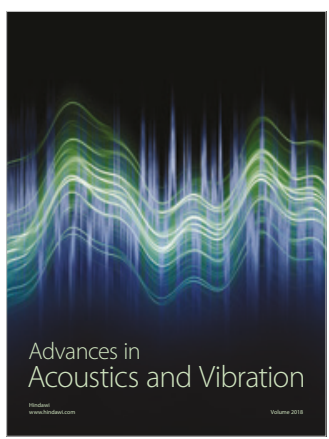

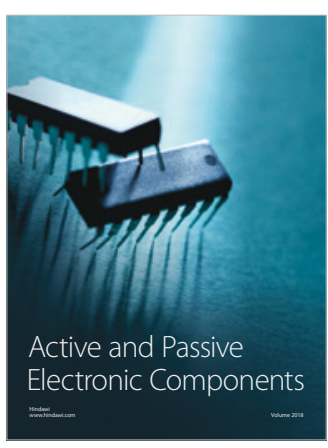
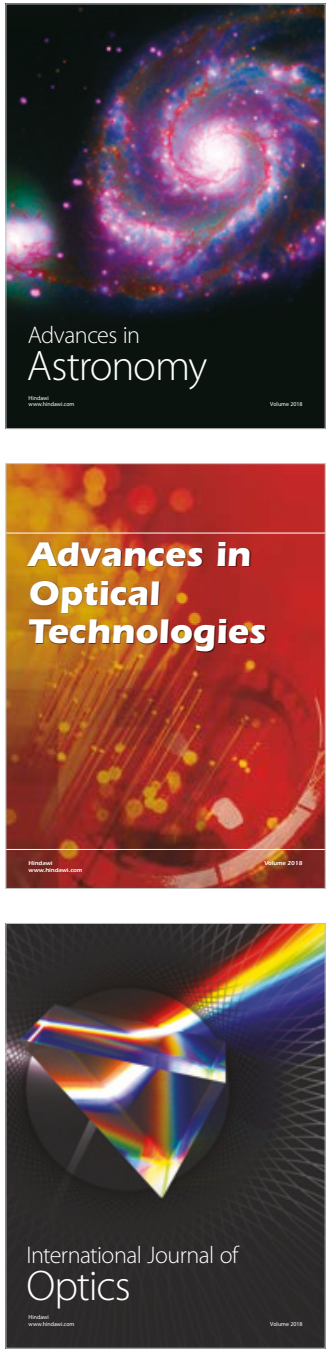